\title{
THE NUMBER OF SEMIGROUPS OF ORDER $n$
}

\author{
DANIEL J. KLEITMAN ${ }^{1}$, BRUCE R. ROTHSCHILD AND JOEL H. SPENCER ${ }^{1}$
}

\begin{abstract}
The number of semigroups on $n$ elements is counted asymptotically for large $n$. It is shown that "almost all" semigroups on $n$ elements have the following property: The $n$ elements are split into sets $A, B$ and there is an $e \in B$ so that whenever $x, y \in A, x y \in B$, but if $x$ or $y$ is in $B, x y=e$.
\end{abstract}

1. The problem. Fix a labelled $n$-element set $[n]=\{1, \ldots, n\}$. A semigroup $S G$ on $[n]$ is an associative binary operation (denoted by concatenation). Let $S(n)$ denote the number of semigroups on $[n]$. We find an asymptotic approximation to $S(n)$. Let

$$
f(t)=\left(\begin{array}{c}
n \\
t
\end{array}\right) t^{1+(n-t)^{2}}
$$

\section{THEOREM 1.}

$$
S(n)=\left[\sum_{t=1}^{n} f(t)\right](1+o(1))
$$

Define $t_{0}=t_{0}(n)$ as that $t$ which maximizes $f(t)$. One can show

$$
t_{0} \sim n /(2 \ln n)
$$

and $f(t)$ has a sharp peak at $t_{0}$. Equation (1.2) simplifies to

$$
S(n)=f\left(t_{0}\right)(1+o(1))
$$

except in those "rare" instances when $f\left(t_{0}+1\right)$ or $f\left(t_{0}-1\right)$ are "near" $f\left(t_{0}\right)$.

For the remainder of the paper we adopt the convention that any inequality about functions of $n$ is meant to be true only for all $n$ sufficiently large, where how large depends on the statement.

2. Construction of the semigroups. Let $A \subseteq[n],|A|=n-t, t \geqq 1$. Set $B=A^{0}$. We construct a family $\delta(A)$ of semigroups on $[n]$ as follows:

(i) Select $e \in B$.

(ii) For $x, y \in A$ define $x y$ to be an arbitrary member of $B$.

(iii) For $x \in B$ or $y \in B$ define $x y=e$.

Received by the editors May 11, 1973.

AMS (MOS) subject classifications (1970). Primary 05A15, $20 \mathrm{M} 99$.

Key words and phrases. Semigroup, asymptotic enumeration.

${ }^{1}$ Research supported by ONR N00014-67-A-0204-0063 and NSF GP-33580X. 
Then for all $x, y, z \in[n], x y \in B$ so $(x y) z=e$. Similarly, $x(y z)=e$. This yields

$$
|\delta(A)|=t^{1+(n-t)^{2}}
$$

semigroups. Call a semigroup $S G$ of type $\mathrm{T}$ (for trivial) if it is of the above form for some $A, e$.

Let $\delta_{1}(A)$ denote those semigroups $S G \in \delta(A)$ such that for no $a \in A$ is $a x$ $=x a=e$ for all $x \in[n]$. Then

$$
\left|\delta_{1}(A)\right|=(1+o(1))|\delta(A)|
$$

and the $\delta_{1}(A)$ are disjoint. Hence, there are $\left(\sum_{t=0}^{n} f(t)\right)(1+o(1))$ semigroups of type $\mathrm{T}$. This implies

$$
S(n) \geqq\left[\sum_{t=0}^{n} f(t)\right](1+o(1)) .
$$

3. An upper-bound. Let $\sigma(A)$ denote the family of semigroups for which $A$ is a minimal (in cardinality) set of generators. (I.e. all $x \in[n]$ may be expressed as $x=a_{1} \cdots a_{k}$ for some $k \geqq 1, a_{1}, \ldots, a_{k} \in A$.) Set $N(A)$ $=|\Im(A)|$. Then

$$
S(n) \leqq \sum_{A \subseteq[n]} N(A)
$$

If $a_{1}, a_{2} \in A$ then

$$
a_{1} a_{2} \in\left\{a_{1}, a_{2}\right\} \cup B,
$$

as otherwise $A-\left\{a_{1} a_{2}\right\}$ would be a smaller set of generators. We bound $N(A)$ by constructing all $S G \in \mathcal{T}(A)$ as follows:

(i) For $a_{1}, a_{2} \in A$ choose $a_{1} a_{2} \in\left\{a_{1}, a_{2}\right\} \cup B$. (This may be done in $(t+2)^{(n-t)^{2}}$ ways.)

(ii) Let $A^{\prime}$ be a minimal (in cardinality) subset of $A$ so that $A^{\prime} A \supseteq A A \cap B$. For each $b \in A A \cap B$ there exist $a_{b}{ }^{\prime}, a_{b}$ so that $b=a_{b}{ }^{\prime} a_{b}$. The set $X$ $=\left\{a_{b}^{\prime}: b \in B\right\}$ satisfies $X A \supseteq A A \cap B$, so that

$$
\left|A^{\prime}\right| \leqq|X| \leqq|B|=t
$$

Since $A^{\prime} \subseteq A,\left|A^{\prime}\right| \leqq \min (t, n-t)$.

Now for all $a \in A^{\prime}, b \in B$ choose $a b$ arbitrarily. (This may be done in at most $n^{\left|A^{\prime}\right||B|} \leqq n^{t \min (t, n-t)}$ ways.)

Claim 1. $S G$ is determined by $A A \cup A^{\prime} B$.

Proof. As all $x, y \in[n]$ are finite products of $A$ 's, it suffices to show that all $a_{1} a_{2} \cdots a_{k}$ are determined. We show this by induction, it being trivial for $k \leqq 2$. For $k>2$ set $a_{1} a_{2}=b$. If $b \in A, a_{1} a_{2} \cdots a_{k}=b a_{3} \cdots a_{k}$ is determined by induction. If $b \notin A, b \in B \cap A A$ so $b=c d$ for some $c \in A^{\prime}, d$ $\in A$. Then $a_{1} \cdots a_{k}=c\left(d a_{3} \cdots a_{k}\right)$. Now $x=d a_{3} \cdots a_{k}$ is determined by induction and $c x$ is determined since $A^{\prime}[n]$ is determined.

We have shown 


$$
N(A) \leqq(t+2)^{(n-t)^{2}} n^{t \min (t, n-t)} .
$$

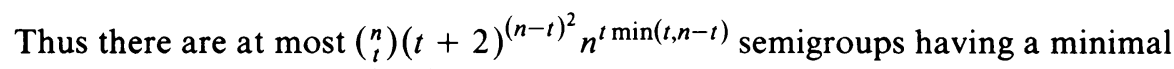
set of generators of cardinality $t$.

From (3.1), (3.4),

$$
S(n) \leqq \sum_{t=1}^{n}\left(\begin{array}{l}
n \\
t
\end{array}\right)(t+2)^{(n-t)^{2}} n^{t \min (t, n-t)}
$$

so that

$$
S(n)=[n /(2 e+o(1)) \ln n]^{n^{2}} .
$$

Let $\varepsilon>0$ be fixed and small $\left(\varepsilon=10^{-6}\right.$ will do). By elementary calculations and (2.3),

$$
\Sigma^{\prime}\left(\begin{array}{c}
n \\
t
\end{array}\right)(t+2)^{(n-t)^{2}} n^{t \min (t, n-t)}=o(S(n))
$$

where $\Sigma^{\prime}$ runs over $t,\left|t-t_{0}\right|>\varepsilon n / \ln n$. Thus: Almost all semigroups are in T( $A$ ) for some $A,|A|=n-t,\left|t-t_{0}\right| \leqq \varepsilon n / \ln n$. We restrict our attention to $t$ in this range for the duration of this paper.

4. The easy case. Our ultimate objective is to show

$$
N(A)=t^{1+(n-t)^{2}}(1+o(1))
$$

for $|A|=n-t,\left|t-t_{0}\right|<\varepsilon n / \ln n$. In this section we show the corresponding result for a restricted class of semigroups.

We say that a semigroup in $\Im(A)$ has property $\mathrm{E}$ (for easy) if $a_{1}, a_{2}$ $\in A$ imply $a_{1} a_{2} \neq a_{1}$ and $a_{1} a_{2} \neq a_{2}$. Let $\widetilde{T}^{*}(A)$ denote the subclass of $\mathscr{T}(A)$ satisfying property $\mathrm{E}$ and $N^{*}(A)=\left|\sigma^{*}(A)\right|$. We shall show

$$
N^{*}(A)=t^{1+(n-t)^{2}}(1+o(1))
$$

for $|A|=n-t,\left|t-t_{0}\right|<\varepsilon n / \ln n$.

For $S G \in \mathscr{T}^{*}(A)$ set

$$
G_{a x}=\{b \in B: a b=x\} \quad \text { for } a \in A, x \in[n] .
$$

Set

$$
F(a)=\max _{x \in[n]}\left|G_{a x}\right|
$$

and

$$
\delta=t-\min _{a \in A} F(a) .
$$

The number $\delta$ defined above may be thought of as follows. Consider the rows $a B, a \in A$. Each row has a most frequent entry. $\delta$ gives a uniform upper bound (over $a \in A$ ) for the number of entries in $a B$ not equal to the most frequent one. Trivial semigroups have $\delta=0$. Let $\mathfrak{T}_{\delta}^{*}(A)$ be those semigroups in $\mathcal{T}^{*}(A)$ with given $\delta$ and $N_{\delta}^{*}(A)=\left|\mathscr{T}_{\delta}^{*}(A)\right|$. 
We now bound $N_{\delta}^{*}(A)$. The semigroups in $\widetilde{T}_{\delta}^{*}(A)$ may be constructed by the following procedure.

(i) Pick $g \in A$ so that $t-\delta=F(g)$. (This may be done in $n-t \leqq n$ ways.)

(ii) Set $A^{\prime}$ equal a minimal subset of $A$ containing $g$ so that $A^{\prime} A \supseteq A A \cap B$. fix $A^{\prime}$. (Since $\left|A^{\prime}\right| \leqq t$, this may be done in at most $n^{t}$ ways.)

(iii) Determine $A^{\prime} B$. For each $a \in A^{\prime}, F(a) \geqq t-\delta$; so there exists $x \in[n]$, $\left|G_{a x}\right| \geqq t-\delta$. Now $a B$ may be determined by selecting $x$ (in at most $n$ ways), $G_{a x}$ (in $\sum_{i=t-\delta}^{t}\left(\begin{array}{l}t \\ i\end{array}\right) \leqq 1+t^{\delta} \leqq n^{\delta}$ ways), and determining $a b$ for $b \in B$ $-G_{a x}$ (in $n^{|B|-\left|G_{a x}\right|} \leqq n^{\delta}$ ways.) Thus each row has at most $n^{2 \delta+1}$ possibilities, and therefore $A^{\prime} B$ has at most $n^{(2 \delta+1)\left|A^{\prime}\right|} \leqq n^{(2 \delta+1) t}$ possibilities.

(iv) Determine $g A$. For each $a \in A$ we have $g a \in B$ (by property E). There are at most $t^{n-t}$ possible $g A$.

(v) Define an equivalence class on $A$ by $a \equiv b$ if $g a=g b$. Since $g A \subseteq B$ there are at most $t$ equivalence classes and we select representatives $g=a_{1}$, $\ldots, a_{s}, s \leqq t$, of the classes in an arbitrary fashion. For $2 \leqq i \leqq s$ we determine $a_{i} A$, each in at most $t^{n-t}$ ways, for a total of $t^{(n-t)(s-1)}$ possibilities.

(vi) Determine the remainder of $A A$. Let $a, c \in A, a \neq a_{1}, \ldots, a_{s}$. Then $a \equiv a_{i}$ for some $i, 1 \leqq i \leqq s$. Then

$$
g(a c)=(g a) c=\left(g a_{i}\right) c=g\left(a_{i} c\right) \text { and } a c \in G_{g, g\left(a_{i} c\right)} .
$$

Now $a_{i} c$ has already been determined, so $g\left(a_{i} c\right)$ has been determined, as has $G_{g, g\left(a_{i} c\right)}$. From the definition of $\delta$,

$$
\left|G_{g, g\left(a_{i} c\right)}\right| \leqq t-\delta
$$

The product $a c$ can be determined in at most $t-\delta$ ways. This holds for $(n-t)(n-t-s)$ pairs $(a, c)$, giving $(t-\delta)^{(n-t)(n-t-s)}$ possibilities.

By Claim 1 the semigroup is now determined.

$$
\begin{aligned}
N_{\delta}^{*}(A) & \leqq n n^{t} n^{(2 \delta+1) t} t^{(n-t) s}(t-\delta)^{(n-t)(n-t-s)} \\
& \leqq n^{1+(2 \delta+2) t}(1-\delta / t)^{n^{2}(1+o(1))} t^{(n-t)^{2}}
\end{aligned}
$$

and

$$
\sum_{\delta=1}^{t-1} N_{\delta}^{*}(A)=o\left[t^{1+(n-t)^{2}}\right]
$$

by an elementary calculation (using $t \sim n /(2 \ln n)$ ). That is, almost all semigroups in $\mathcal{T}^{*}(A)$ have $\delta=0$. Intuitively, for $\delta \geqq 1, N_{\delta}^{*}(A)=o\left(t^{(n-t)^{2}}\right)$ because for most $a, c \in A$ the product $a c$ can take at most $(t-\delta)$ values versus $t$ in the $\delta=0$ case.

Call an $S G \in \mathcal{T}^{*}(A)$ absurd if $\delta=0$ but is not trivial. Since $\delta=0$ each $a B$, $a \in A$, is constant.

Claim 2. If $S G \in \mathcal{O}^{*}(A)$ and $A B=$ constant, then $S G$ is trivial.

Proof. Say $A B=e$. For any $a \in A$, a $a \in B$ so $a a a=e$. Now $e \notin A$, since, if it were, $A-\{e\}$ would generate $e$ and, therefore, $[n]$, contradicting the minimality of $|A|$. So $e \in B$. Let $b \in B, x \in[n]$. Since $A$ generates $[n], b$ 
$=a_{1} \cdots a_{s}(s \geqq 2), x=a_{s+1} \cdots a_{s+t}(t \geqq 1)$ so $b x=a_{1} \cdots a_{s+t}=e$ as $s$ $+t \geqq 3$. That is, $B[n]=e$ so $S G$ is trivial.

Claim 3. Let $S G \in \mathcal{T}^{*}(A), \delta=0, a_{1}, a_{2} \in A, a_{1} B=e_{1} \neq e_{2}=a_{2} B$. Then $a_{1} A \cap a_{2} A=\varnothing$.

Proof. Suppose $x, y \in A, a_{1} x=a_{2} y$. For any $z \in A, x z, y z \in B$ so $e_{1}$ $=a_{1}(x z)=\left(a_{1} x\right) z=\left(a_{2} y\right) z=a_{2}(y z)=e_{2}$, a contradiction.

The conditions on absurd $S G \in \mathcal{T}^{*}(A)$ imposed by Claim 3 are sufficiently stringent that we may easily show (details omitted) that they are "small" in number (i.e. $o\left(t^{1+(n-t)^{2}}\right)$ ). Hence, almost all $S G \in \mathcal{T}^{*}(A)$ are trivial, yielding (4.2).

5. The general case-outline. Theorem 1 is implied by (4.1). We give a brief outline of the proof of (4.1).

We let $\sigma(A, \delta, \ldots)$ and $N(A, \delta, \ldots)$ denote the set, and number, of semigroups with parameters $A, \delta, \ldots$ By $N(A, \delta, \ldots)$ "small" we always mean in comparison to $t^{(n-t)^{2}}$.

For a particular counting scheme let $\nu(a)$ denote the number of possible rows $a A$ and $\mu(a)=\nu(a) / t^{n-t}$. By (3.2) all $\nu(a) \leqq(t+2)^{n-t}$ so $\mu(a) \leqq n^{4}$.

For $a \in A$ set

$$
S_{a}=\{x \in A: a x=x\} \quad \text { and } L=\left\{a \in A:\left|S_{a}\right| \geqq .01 n\right\}, \quad l=|L| .
$$

If $a \in L$ there are less than $2^{n}$ choices for $S_{a}$ and $n^{.99 n}$ choices for $a\left([n]-S_{a}\right)$. There are less than $2^{n} n^{.99 n}=t^{(.99+o(1)) n}$ choices for $a[n]$. We may determine $S G \in \mathcal{T}(A, L)$ by determining $L A$, then $(A-L) A$, then defining $A^{\prime}$ and determining $A^{\prime} B$. If $l$ is "large", $N(A, L)$ is "small". Most $S G \in \mathcal{T}(A)$ have $l$ $=o(n)$-which we assume for the duration.

We modify the bounding of $N_{\delta}^{*}(A)$ in $\S 4$ by (4.4) to $N(A, L, \delta)$. We need a slightly different definition for $\delta$ :

$$
\delta=t-\min _{a \in A-L} F(a) .
$$

We fix $g \in A-L$ so that $F(g)=t-\delta$. We define $A^{\prime}$ as the minimal set so that $\left(A^{\prime} \cup L\right) A \supseteq A A \cap B$. We bound $N(A, L, \delta)$ by first determining $L[n]$ and then following steps (i), ..., (vi) of $\S 4$. The equivalence classes, in step (v), are defined on $A-L$ and there will be $s \leqq t+1+.01 n$ such classes (when $g a=a,\{a\}$ is an equivalence class). We find

$$
\begin{aligned}
N(A, L, \delta) \leqq & t^{(.99+o(1)) n l} n n^{t} n^{(2 \delta+1) t} \\
& \cdot(t+2)^{s(n-t)}(t+2-\delta)^{(n-t-l-s)(n-t)} .
\end{aligned}
$$

For $\delta \geqq 3, N(A, L, \delta)$ is "small". We assume $\delta \leqq 2$ for the duration. Note this implies $|a B| \leqq 3$ for all $a \in A-L$.

Set

$$
\begin{aligned}
I & =\{a \in A-L: a a=a\}, \\
V & =\{a \in A-L-I: \exists x \in A-L, a \neq x, a x=a \text { or } x a=a\}, \\
|I| & =i|V|=v .
\end{aligned}
$$


For $a \in I, x \in A-\{a\}$,

$$
a x=(a a) x=a(a x) \in\{a, x\} \cup a B,
$$

at most 5 possibilities, so $\nu(a) \leqq 5^{n-t}, \mu(a)=t^{n(-1+o(1))}$.

Suppose $a \in V, x \in A-L, a x=a$ or $x a=a$ and $x A$ has already been determined.

Case 1. $x a=a$. For all $c \in A$,

$$
a c=(x a) c=x(a c) \in\{x a, x c\} \cup x B
$$

at most 5 possibilities. Then $\nu(a) \leqq 5^{n-t}, \mu(a) \leqq t^{n(-1+o(1))}$.

Case 2. $a x=a$. For $c \in A, x c \neq c$ we have

$$
a c=(a x) c=a(x c) \in\{a x\} \cup a B,
$$

at most 4 possibilities. We determine $a B$ (at most $n^{t}$ ways), then $a A$. Since $x \notin L$, at most $.01 n c$ 's have $x c=c$. For these there are $n$ possible ac. Then $\nu(a) \leqq n^{t} 5^{n} n^{.01 n}=n^{n(.01+o(1))}$, so $\mu(a) \leqq t^{n(-.99+o(1))}$.

If $i$ is large we may bound $N(A, I)$ by determining $I A$, then $(A-I) A$, then $A^{\prime} A$. We assume $i=o(n)$ for the duration.

If $v$ is large we may bound $N(A, V)$ by determining $(A-V) A$, then $V A$, then $A^{\prime} A$. (A technical problem arises. For $a \in V, a x=a$ or $x a=a$, we want to determine $a A$ after $x A$. But perhaps $x \in V$. One may order $V$ (trying any ordering and its reverse) so at least half the $a \in V$ come after their respective $x$.) We may assume $v=o(n)$ for the duration.

We bound $N(A, L, I, V, \delta)$, where $l, i, v=o(n)$. We determine $x A$ for $x$ $\in L \cup I \cup V$-and when $x A$ is needed before $a A, a \in V$. We define equivalence classes on the remaining $x \in A$, determining $x A$ first for $x$ a representative, then for the remainder of $A$. We achieve an expression analogous to (5.2) with a factor of $(t-\delta)^{m(n-t)}$ where $m$, the number of "remaining" $A$, is at least $(.99+o(1)) n$. The expression is "small" for $\delta>0$. We assume $\delta=0$. If $L \cup I \cup V \neq \varnothing$ we have factors $\mu(a) \leqq t^{(-.99+o(1)) n}$ that are not adequately counterbalanced so that $N(A, \delta, L, I, V)$ is "small". Most semigroups have $\delta=0, L=I=V=\varnothing$ and are trivial.

(A final note on "filling in details". One shows $N(A, \delta, L, \ldots)$ $\leqq t^{-.99 n} t^{(n-t)^{2}}$ and there are less than, say, $5^{n}$ possible $\delta, L, \ldots$ so that $\sum N(A, \delta, L, \ldots)$ taken over all $\delta, L, \ldots$, except $\delta=0, L=\cdots=\varnothing$, is small.)

Department of Mathematics, Massachusetts Institute of Technology, Cambridge, MASSACHUSETTS 02139 (Current address of Bruce Rothschild)

Department of Mathematics, University of California, los Angeles, California 90024 (Current address of Daniel Kleitman)

Current address: (Joel Spencer): Department of Mathematics, State University of New York at Stony Brook, Stony Brook, Long Island, New York 11794 